\title{
Toluidine blue staining reveals changes in chromatin stabilization of mouse spermatozoa during epididymal maturation and penetration of ova
}

\author{
Halina Krzanowska \\ Department of Genetics and Evolution, Institute of Zoology, Jagiellonian University, Karasia 6, \\ 30-060 Kraków, Poland
}

\begin{abstract}
Summary. Sperm samples, recovered from various regions of the reproductive tract of male and female mice, were air dried, fixed for $2 \mathrm{~min}$ in acetic alcohol and stained with toluidine blue. Testicular sperm heads were deeply stained, while in the samples from the successive regions of caput epididymidis the proportion of stained heads gradually decreased and many of them were stained in their distal part only. Spermatozoa from the corpus and cauda epididymidis, vas deferens, uterus, oviduct and from the perivitelline space were colourless (except for one type of misshapen head). Sperm heads that had penetrated the vitellus became stained distally. Vas deferens sperm heads were fully stained after treatment with dithiothreitol for $30 \mathrm{~min}$. We suggest that the inability to stain with toluidine blue is characteristic of sperm chromatin stabilized by disulphide bonds.
\end{abstract}

\section{Introduction}

During our experiments of in-vitro fertilization of mouse ova it was noticed that sperm heads that had penetrated the ooplasm stained deeply with toluidine blue, even before chromatin decondensation, while the surrounding spermatozoa remained colourless ( $\mathrm{H}$. Krzanowska \& $\mathrm{E}$. Lorenc, unpublished). The probable explanation of this difference was that toluidine blue staining might reveal changes in chromatin stabilization. It is known that during the final maturation of mammalian spermatozoa in the epididymis sperm chromatin undergoes stabilization through the formation of disulphide cross-links (Calvin \& Bedford, 1971), a process that should be reversed at fertilization.

The ability of spermatozoa to stain with toluidine blue has been investigated in the present study in relation to chromatin stabilization of normal and misshapen spermatozoa recovered from different regions of the reproductive tract of male and female mice.

\section{Materials and Methods}

Sperm samples from the reproductive tract of the male

Mature, 3-month-old males of the inbred mouse strains, $\mathrm{CBA} / \mathrm{Kw}, \mathrm{C} 57 \mathrm{BL} / \mathrm{Kw}$ and $\mathrm{KE}(5$ animals of each strain) and two 7-week-old $\mathrm{KE}$ males were killed by cervical dislocation and their reproductive organs were cut under the dissecting microscope at the levels indicated in Text-fig. 1 (starting from the vas deferens).

Untreated preparations. Vas deferens, cauda, corpus and three parts of the caput epididymidis, and the testis were transferred to separate drops of saline $(0.95 \%(\mathrm{w} / \mathrm{v}) \mathrm{NaCl})$ and 
cut into pieces. Small drops of the sperm suspension released from each region were spread on slides and air dried.

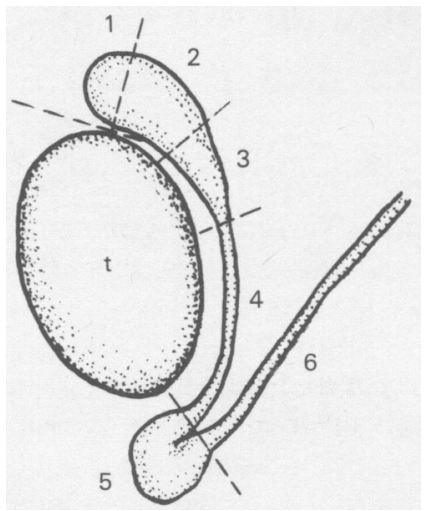

Text-fig. 1. Regions of the male reproductive organs from which sperm samples were obtained; $t$, testis; $1-3$, caput epididymidis; 4 , corpus epididymidis; 5 , cauda epididymidis; 6 , vas deferens; --- levels of cutting.

Incubated preparations. The remaining sperm samples from the vas deferens and cauda epididymidis were suspended in $0.5 \mathrm{ml}$ saline. Aliquots $(0.1 \mathrm{ml})$ were mixed with equal amounts of $0.1-0.0001 \mathrm{M}$ dithiothreitol (Loba Chemie, Wien-Fischamend) in $0.95 \%(\mathrm{w} / \mathrm{v}) \mathrm{NaCl}$, incubated at room temperature $\left(\sim 22^{\circ} \mathrm{C}\right)$ for $30 \mathrm{~min}$ and centrifuged at $200 \mathrm{~g}$ for $5 \mathrm{~min}$. The pellets were resuspended in $1.0 \mathrm{ml}$ saline and centrifuged; the dense sperm suspension was spread on slides and air dried.

\section{Samples from the reproductive tract of the female}

Ten KE females were killed 2-3 h after copulation with adult KE males. The oviducts, severed from the ovaries and at a point directly above the uterotubal junction, were placed in a drop of saline. The ampullar wall of the left oviduct was torn open with a needle, allowing the ova within their cumulus masses to flow out onto the slide and dry in air (ampullary contents). The ova recovered from the right oviduct were treated with $0.025 \%$ hyaluronidase for the dispersion of granulosa cells, then washed with saline and inspected under the microscope. Any ova containing free spermatozoa under the zona pellucida or a sperm head penetrated into the vitellus (sperm tail seen in the perivitelline space) were separately transferred onto the slides (with a minimal amount of fluid) and air dried.

The rest of the oviduct was flushed from the uterine end with a small amount of saline and the oviducal contents were air dried on the slide. Finally, a drop of fluid from the uterus was smeared on the slide and air dried.

\section{Staining and analysis of spermatozoa}

The preparations were fixed in acetic alcohol ( 3 parts absolute ethanol, 1 part glacial acetic acid) for $2 \mathrm{~min}$. Fixation for $>5 \mathrm{~min}$ tended to reduce the staining ability of spermatozoa, but not of spermatids and of sperm heads inside the vitellus. After fixation the slides were air dried, stained with $1 \%(\mathrm{w} / \mathrm{v})$ aqueous toluidine blue for $15 \mathrm{~min}$, washed with distilled water, covered with coverslips and inspected under the microscope. The percentages of stained sperm heads, normal and abnormal, were calculated from 100 spermatozoa per preparation when possible. The classification of abnormal sperm heads was based on previous schemes (Krzanowska, 1976, 1981). To ascertain the correct classification of colourless spermatozoa, the preparations from 
the female reproductive tract were stained with Feulgen (after removal of coverslips under water) and again examined.

\section{Untreated preparations}

\section{Results}

The heads of spermatids and spermatozoa released from the testes were deeply stained (PI. 1 Fig. 1), with the exception of severely abnormal 'thread-like heads' (Class 5), seen only in young males. The samples from the cauda epididymidis contained completely or partly stained and unstained sperm heads. The characteristic feature of those partly stained was the dark distal part and the colourless apical part; the stained area varied in size, sometimes being limited to the narrow strip at the base of the head (PI. 1, Figs 2-4). The percentages of unstained sperm heads rose from low values for region 1 of the caput epididymidis to nearly 100\% for region 3 (Table 1). These percentages are to be taken as approximate because the levels of cutting could not be estimated precisely, but the same trend was found for every male.

Normal and misshapen (Classes 1, 3,4 and 5) spermatozoa from the distal parts of the male reproductive tract (corpus and cauda epididymidis, vas deferens) and from the uterus were always colourless. However, about $50 \%$ of Class 2 sperm heads (abnormals amounting to $9 \%$ in the KE strain males; Krzanowska, 1981) was faintly or deeply stained, especially in the vicinity of the 'canal' or slit inside the sperm nucleus (P1. 1, Fig. 5).

Table 1. Staining ability of sperm heads from 3 regions

(Text-fig. 1) of the mouse caput epididymidis

\begin{tabular}{clcl}
\hline & \multicolumn{3}{c}{ Percentages of sperm heads* } \\
\cline { 2 - 3 } Region & \multicolumn{2}{c}{ Stained } \\
\cline { 2 - 4 } & Completely & Partly & Unstained \\
\hline 1 & $44(10-75) \dagger$ & $40(11-60)$ & $16(0-45)$ \\
2 & $10(0-21)$ & $38(11-83)$ & $52(14-82)$ \\
3 & $1(0-2)$ & $2(0-2)$ & $97(96-100)$ \\
\hline
\end{tabular}

* Excluding Class 2 and 5 abnormal spermatozoa.

$\uparrow$ Mean (range) on preparations from 17 males.

In the oviducal and ampullary contents the proportion of Class 2 and 3 abnormal spermatozoa was greatly reduced $(2.6$ and 1.7 -in contrast to 9.1 and $6.2 \%$ in the uterus, respectively) and there were no Class 4 abnormal spermatozoa. Of the 1500 sperm heads scored all were colourless, except for 3 stained Class 2 abnormals, one of which was seen inside the layer of granulosa cells. Only normal unstained heads were found in the perivitelline space (18 scored).

However, sperm heads that had penetrated into the vitellus and were at different stages of chromatin decondensation were always stained. Of the two which still had condensed chromatin, one was completely stained and one in its distal part only (see Pl. 1, Fig. 6). The same staining pattern was seen in many spermatozoa which had penetrated the vitellus after insemination in vitro (H. Krzanowska \& E. Lorenc, unpublished). (However, for a clear preparation of the whole ovum, 30 min fixation is recommended; it does not deteriorate the staining ability of sperm heads inside the vitellus.)

\section{Incubated preparations}

In the samples treated with $0.1 \mathrm{M}$-dithiothreitol (the final concentration being $0.05 \mathrm{M}$ ) all sperm heads were deeply stained (P1. 1, Fig. 5, inset), except for those of Class 5. Chromatin 
decondensation, seen at the margins of many preparations, occurred probably during or after drying, because in the drops inspected just after centrifugation all spermatozoa seemed to be intact. The preparations treated with $0.01 \mathrm{M}$-dithiothreitol contained $50-100 \%$ completely stained heads and $0-50 \%$ of partly stained, but without any regular pattern. The unstained area was confined to the apical, distal or central part of the head. No differences between strains of mice were detected. After $0.001 \mathrm{M}$-dithiothreitol treatment sperm heads were colourless (or uniformly slightly bluish), except for a few that stained more darkly, among them Class 2 abnormals. The samples treated with 0.0001 m-dithiothreitol did not differ from untreated samples.

\section{Discussion}

It has been reported (Gledhill, 1971) that the changes occurring in the nucleoprotein complex of mammalian germ cells during spermateliosis are accompanied by a marked reduction of the reactivity to Feulgen staining and by a decrease in the number of nuclear binding sites for basic dyes (e.g. acridine orange and methyl green). During epididymal maturation of spermatozoa a further reduction in Feulgen staining ability was observed. This appears to be the result of chromatin stabilization by disulphide bonds, because stainability is reactivated after dithiothreitol treatment (Esnault, 1973). The present results show that the changes in chromatin stabilization are very clearly revealed by the simple method of toluidine blue staining which gives positive reaction only with unstabilized chromatin. The characteristic staining properties of spermatozoa from the caput epididymidis confirm the ordered pattern of sperm chromatin stabilization from the apical to the distal part of the sperm head, as first suggested by Calvin \& Bedford (1971) in their studies on sperm decondensation in vitro. On the other hand, the lack of any regular staining pattern in dithiothreitol-treated spermatozoa supports the view of these authors that the -S-S- bonds are evenly distributed in mature sperm heads.

Bedford \& Calvin (1974) consider that mammalian sperm head stabilization through disulphide cross-links has evolved as a structural adaptation for penetration of the thick and compact zona pellucida. The observation that supplementary spermatozoa attached to fertilized eggs could not be lysed by SDS alone (Calvin \& Bedford, 1971) led to the conclusion that reduction of the $-\mathrm{S}-\mathrm{S}$ - linkages should occur following penetration of the vitellus. This conclusion is supported by our results with toluidine blue staining: normal sperm heads recovered from the female reproductive tract and from the perivitelline space were always colourless, but acquired the staining ability after penetration into the ooplasm.

Gledhill (1975) noted that "the pattern of ultrastructural change seen during decondensation of the chromatin to form a pronucleus may be a programmed phenomenon which reverses the nuclear alterations of spermiogenesis." The same appears to apply to the changes in chromatin stabilization, as illustrated by a striking similarity of the staining pattern of sperm heads after penetration (Pl. 1, Fig. 6, inset) and those in the caput epididymidis (Pl. 1, Fig. 4). However, in contrast to epididymal spermatozoa, sperm heads inside the vitellus (like testicular spermatids) do not lose their staining ability after prolonged fixation, and this suggests that the reduction of disulphide bonds after penetration is immediately followed by further alterations of chromatin proteins.

The staining ability of misshapen sperm neads was like that of normal heads, except for those in Class 5 which were always colourless (also Feulgen negative, probably devoid of chromatin; Krzanowska, 1981) and in Class 2 which were often stained even in the ejaculated samples. Many heads of Class 2 spermatozoa stain violet or reddish (instead of green) with methyl green-pyronin (Krzanowska, 1976), as do stage 13 spermatids, and show only a weak incorporation of arginine (Godowicz, 1977). This suggests that Class 2 abnormal spermatozoa do not pass through the final steps of chromatin maturation during spermiogenesis, which may 
PLATE 1

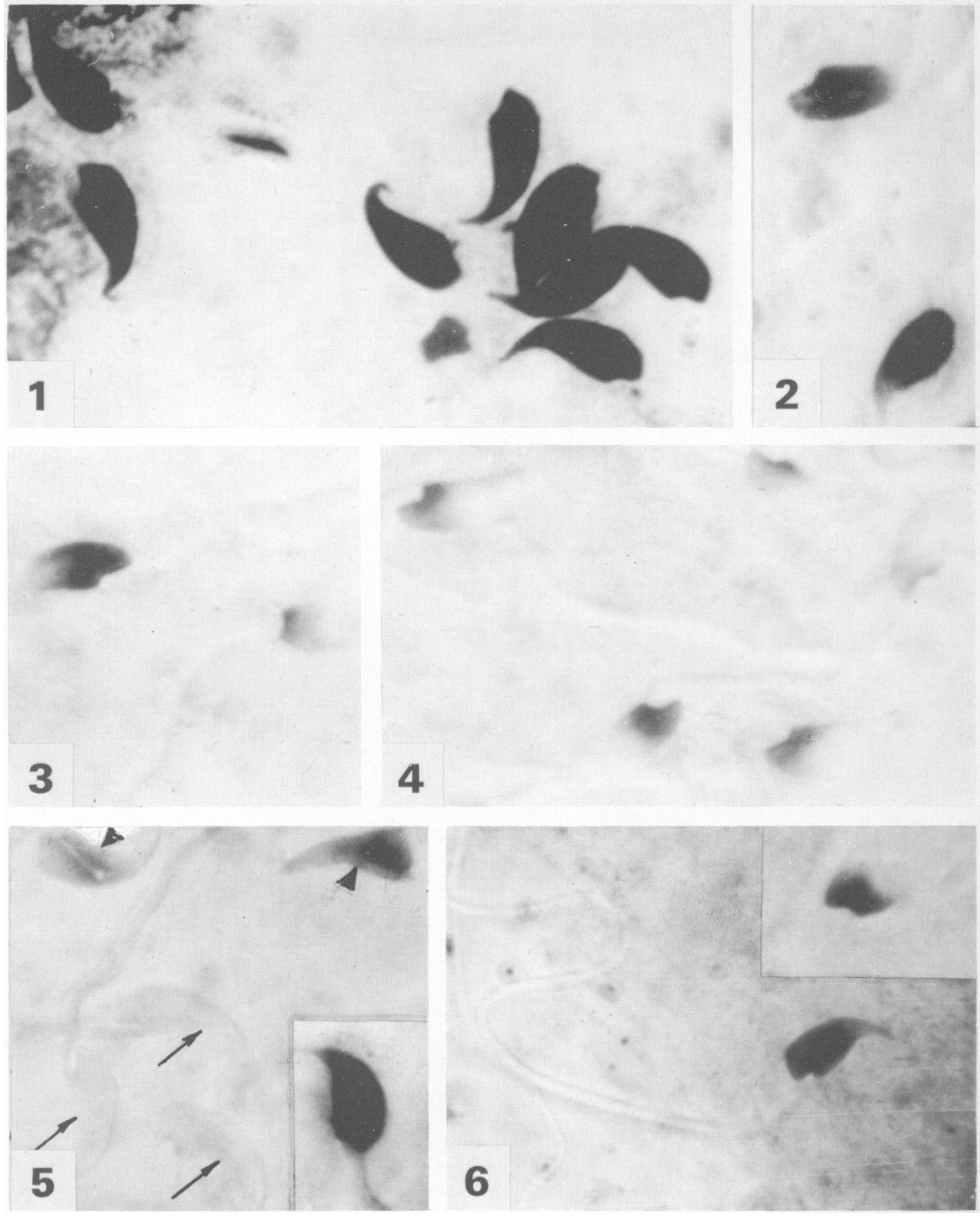

Toluidine blue-stained mouse spermatozoa.

Fig. 1. Deeply stained sperm heads from the testis. $\times 2500$.

Figs 2-4. Partly stained sperm heads from the caput epididymidis. $\times 2500$.

Fig. 5. Spermatozoa from the vas deferens. Normal heads unstained (arrows), misshapen Class 2 heads stained (arrowheads). Inset: deeply stained head of a normal spermatozoon incubated in $0 \cdot 1$ M-dithiothreitol. $\times 2500$.

Fig. 6. One completely and one partly (inset) stained sperm head inside the vitellus, after in-vitro insemination. $\times 1900$; inset $: \times 2500$.

(Facing p. 100) 
later result in deficient $-\mathrm{S}-\mathrm{S}-$ bonding. The formation of certain misshapen sperm heads in bulls involves some defects in binding nuclear protein to DNA during spermateliosis (Gledhill, Darżynkiewicz \& Ringertz, 1971). Gledhill (1971) speculated that abnormal heads with less stable chromatin could be more susceptible to the action of lytic substances and macrophages and consequently could be preferentially removed in the excurrent male duct system. Godowicz (1979) found that the proportion of Class 2 abnormal spermatozoa was significantly lower in the vas deferens than in the caput epididymidis of the KE males, but in C57 males the same was true of Class 3 abnormals which do not differ in staining ability from the normal heads. Above the uterotubal junction the proportion of abnormal sperm heads was greatly reduced (this study and Krzanowska, 1974) and stained Class 2 abnormal spermatozoa were so rarely seen that their preferential removal seems likely.

No strain differences were noticed in toluidine blue staining ability for normal untreated or dithiothreitol-incubated spermatozoa, although the resistance of mammalian sperm heads to the action of decondensing agents has been shown to vary between species (Mahi \& Yanagimachi, 1975; Delgado, Huacuja, Merchant, Reyes \& Rosado, 1980) and a difference between inbred and outbred mice has been reported (Reid, 1977).

This work was supported by the Polish Academy of Sciences within the project MR II-9.

\section{References}

Bedford, J.M. \& Calvin, H.I. (1974) The occurrence and possible functional significance of -S-S- crosslinks in sperm heads, with particular reference to eutherian mammals. J. exp. Zool. 188, 137-156.

Calvin, H.I. \& Bedford, J.M. (1971) Formation of disulphide bonds in the nucleus and accessory structures of mammalian spermatozoa during maturation in the epididymis. J. Reprod. Fert., Suppl. 13, 65-75.

Delgado, N.M., Huacuja, L., Merchant, H., Reyes, R. \& Rosado, A. (1980) Species specific decondensation of human spermatozoa nuclei by heparin. Arch. Androl. 4, 305-318.

Esnault, C. (1973) Reactivation of the Feulgen reaction of ram spermatozoa by dithiothreitol. J. Reprod. Fert. 32, 153-157.

Gledhil,, B.L. (1971) Changes in deoxyribonucleoprotein in relation to spermateliosis and the epididymal maturation of spermatozoa. J. Reprod. Fert., Suppl. 13, 77-88.

Gledhill, B.L. (1975) Nuclear changes during mammalian spermiogenesis. In The Biology of the Male Gamete (Eds J. G. Duckett \& P. A. Racey). Biol. J. Linnean Soc. 7, Suppl. 1, 215-226.

Gledhill, B.L., Darżynkiewicz, \& Ringertz, N.R. (1971) Changes in deoxyribonucleoprotein during spermio- genesis in the bull: increased ${ }^{3} \mathrm{H}$-actinomycin $\mathrm{D}$ binding to nuclear chromatin of morphologically abnormal spermatozoa. J. Reprod. Fert. 26, 25-28.

Godowicz, B. (1977) Histochemical and autoradiographic studies of abnormal spermatozoa in mice of the inbred KE strain. Folia Histochem. Cytochem. 15, 211-215.

Godowicz, B. (1979) Percentage of abnormal spermatozoa in different parts of the reproductive tracts of the male mice of inbred C57 and KE strains. Zwierzęta Lab. 16, 3-8.

Krzanowska, H. (1974) The passage of abnormal spermatozoa through the uterotubal junction of the mouse. J. Reprod. Fert. 38, 81-90.

Krzanowska, H. (1976) Types of sperm-head abnormalities in four inbred strains of mice. Acta biol. cracov. (Ser. Zool.) 19, 79-85.

Krzanowska, H. (1981) Sperm head abnormalities in relation to the age and strain of mice. $J$. Reprod. Fert. 62, 385-392.

Mahi, C.A. \& Yanagimachi, R. (1975) Induction of nuclear decondensation of mammalian spermatozoa in vitro. J. Reprod. Fert. 44, 293-296.

Reid, B.L. (1977) Protamines in the sperm heads of inbred and outbred mice. J. Reprod. Fert. 49, 169-171.

Received 4 March 1981 